\title{
New method for measuring fluorescence microscopy illumination
}

$\overline{\text { www.CoolLED.com }}$

\begin{abstract}
The intensity of microscope illumination systems has a significant impact on the brightness and contrast of images and therefore data quality. Optimal illumination has been surrounded by complex, confusing and sometimes inaccurate measurements and terminology, but a new method is putting a stop to this.
\end{abstract}

\section{INTRODUCTION}

LED illumination systems for fluorescence microscopy present a modern alternative to mercury and metal halide lamps, affording a range of benefits in terms of cost, functionality and the environment. They are now recognised as a mature technology, but one topic lags behind. The industry has not yet settled on a standardised approach for measuring intensity. Without a quantitative baseline, it is impossible to normalise performance requirements and configure system improvements.

We propose correct terminology alongside an industry standard methodology for microscopists to easily quantify the intensity where they need it, at the sample plane. These meaningful data will allow direct comparison of all illumination systems and microscope configurations, allowing scientists to optimise their system to suit their needs.

\section{WHAT ARE WE MEASURING?}

When product specifications or discussions surrounding fluorescence microscopy illumination mention illumination intensity, it is not often clear exactly what this refers to. Firstly, let us clarify what is meant by the term intensity:

- Intensity: Power per unit area. It can refer to any kind of power (electrical, heat, light etc.) and is measured in $\mathrm{W} / \mathrm{m}^{2}$.

- Radiant intensity: Not to be confused with intensity, this measures power per unit steradian (i.e. power per unit cone angle of emission) in W/sr.

- Power: Measured in W, this value is largely meaningless since it does not consider how effectively power is focused onto a given surface area.

- Irradiance: The radiant power at a surface per unit area, measured in $\mathrm{mW} / \mathrm{mm}^{2}$. This is our preferred term and eliminates any confusion between intensity and radiant intensity.

\section{WHERE TO MEASURE IRRADIANCE?}

Some manufacturers quote power from the LED chip, which provides the most impressive sounding statistic. Others might quote light at the end of a liquid light guide. However, these numbers are misleading since they are not representative of the light reaching the sample (Figure 1).

The only significant light is that hitting the sample to excite the fluorophore and the only meaningful place to measure the optical power of an illumination system is therefore at the sample plane. Measuring this irradiance in $\mathrm{mW} / \mathrm{mm}^{2}$ provides accurate values which can form a baseline for the comparison of microscope configurations and illumination systems.

\section{A PROTOCOL FOR MEASURING IRRADIANCE}

At CoolLED we quote irradiance values at the sample plane of a typical fluorescence microscope, for example an Olympus BX51 microscope with a 10x (0.3 NA) or 40x (0.85 NA) objective. 


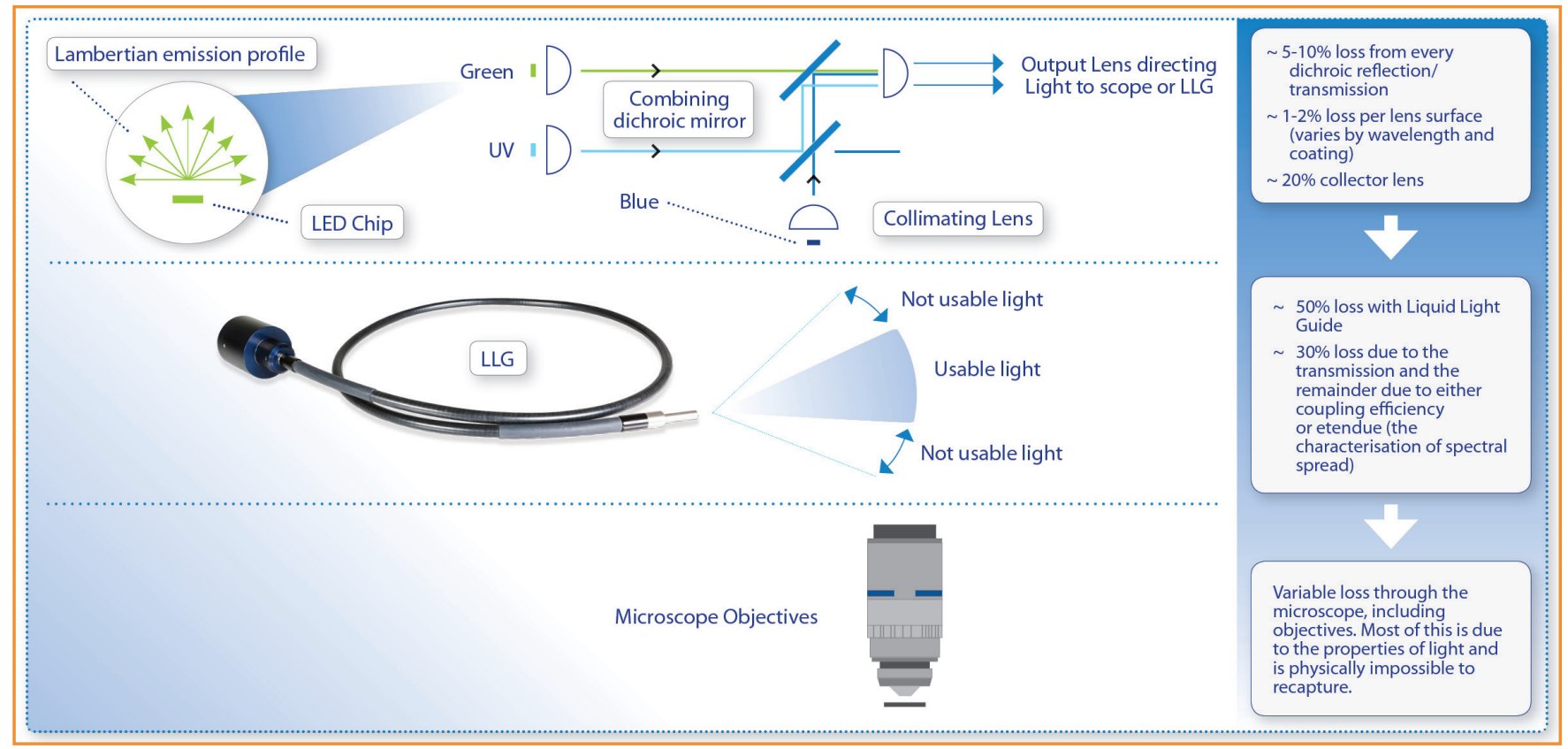

Figure 1: Why measure irradiance at the sample plane. As light travels from the LED to the sample, a portion is lost at each step, for example the Liquid Light Guide (LLG). Measuring light at any point upstream of the sample plane will therefore result in misleading data.

1. Remove the excitation filter from the cube and replace the dichroic mirror with an $100 \%$ mirror. This means we are measuring light at the sample plane unaffected by optical filters.

2. When measuring power at the sample plane, use a calibrated optical spectrometer. We also use an integrating sphere or cosine corrector to acquire a power result that is independent of the ray's angle of incidence on the detector.

3. Close the microscope field stop until the field stop image on the sample plane is just outside the field of view. This ensures that light hitting the sample plane will only consist of light rays that are within the field of view for the objective in use, as seen by an eyepiece or camera with a given field number. This removes light that could be escaping from the objective but not making it into the field of view, achieving a more precise measurement by removing any variability caused from the field stop.

4. Calculate irradiance from the power reading using the surface area at the sample plane:

a. Determine the diameter in $\mathrm{mm}=$ Field number ( $\mathrm{fn}$ ) / objective magnification (Mo).

b. Calculate surface area in $\mathrm{mm}^{2}$ using the equation $\pi \mathrm{r}^{2}$.

c. Use the surface area and power reading to express irradiance in $\mathrm{mW} / \mathrm{mm}^{2}$.

\section{SUMMARY}

As LED illumination technology has evolved, so has the discussion surrounding what is commonly referred to as 'illumination intensity'. The confusion stemming from varying terminology and measurement techniques has created a barrier to identifying which systems truly deliver the most light to the sample plane. We now therefore strongly recommend the use of meaningful irradiance values measured at the sample plane using our standardised protocol, to both compare and boost irradiance when required.

LED irradiance is continuing to improve and we are confident this trend will continue in the coming years. We look forward to enabling the microscopy community to independently monitor these developments with a robust and standardised approach for quantifying irradiance.

For more information, visit www.CoolLED.com

Cool[局D

Simply Better Control

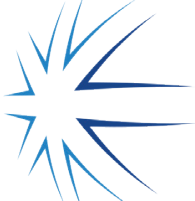

